\title{
Distinct lung cancer subtypes associate to distinct drivers of tumor progression
}

\author{
Valeria Relli ${ }^{1}$, Marco Trerotola ${ }^{1,2}$, Emanuela Guerra ${ }^{1,2}$ and Saverio Alberti ${ }^{1,3}$ \\ 1Unit of Cancer Pathology, CeSI-MeT, University "G. d'Annunzio", Chieti, Italy \\ ${ }^{2}$ Department of Medical, Oral and Biotechnological Sciences, University "G. d'Annunzio", Chieti, Italy \\ ${ }^{3}$ Department of Biomedical Sciences, Dentistry, Morphological and Functional Imaging, University of Messina, Messina, Italy \\ Correspondence to: Saverio Alberti, email: salberti@unime.it \\ Keywords: non-small cell lung cancer; lung adenocarcinomas; lung squamous cell carcinomas; prognostic determinants; survival \\ curves \\ Received: August 31, $2018 \quad$ Accepted: September 26, $2018 \quad$ Published: October 30, 2018 \\ Copyright: Relli et al. This is an open-access article distributed under the terms of the Creative Commons Attribution License 3.0 \\ (CC BY 3.0), which permits unrestricted use, distribution, and reproduction in any medium, provided the original author and source \\ are credited.
}

\section{ABSTRACT}

The main non-small-cell lung cancer (NSCLC) histopathological subtypes are lung adenocarcinomas (LUAD) and lung squamous cell carcinomas (LUSC). To identify candidate progression determinants of NSCLC subtypes, we explored the transcriptomic signatures of LUAD versus LUSC. We then investigated the prognostic impact of the identified tumor-associated determinants. This was done utilizing DNA microarray data from 2,437 NSCLC patients. An independent analysis of a case series of 994 NSCLC was conducted by next-generation sequencing, together with gene expression profiling from GEO (https://www.ncbi.nlm.nih.gov/geo/).

This work led us to identify 69 distinct tumor prognostic determinants, which impact on LUAD or LUSC clinical outcome. These included key drivers of tumor growth and cell cycle, transcription factors and metabolic determinants. Such disease determinants appeared vastly different in LUAD versus LUSC, and often had opposite impact on clinical outcome. These findings indicate that distinct tumor progression pathways are at work in the two NSCLC subtypes. Notably, most prognostic determinants would go inappropriately assessed or even undetected when globally investigating unselected NSCLC. Hence, differential consideration for NSCLC subtypes should be taken into account in current clinical evaluation procedures for lung cancer.

\section{INTRODUCTION}

Lung cancer is traditionally classified as nonsmall-cell lung cancer (NSCLC) and small-cell lung cancer (SCLC) [1]. The two cancer types differ in histopathological traits, genetic changes, prognosis and response to therapy [1]. However, while the usefulness of distinguishing NSCLC from SCLC is clear, far less clear is the reason for jointly categorizing distinct NSCLC subtypes. NSCLC is the most common type of lung cancer, with a poor response to chemotherapy and a low survival rate. This unfavorable treatment response stems from both late diagnosis and from complex, incompletely understood biology. The two main NSCLC histopathological subtypes are lung adenocarcinomas (LUAD) and lung squamous cell carcinomas (LUSC). To define the contribution of major cellular pathways to the biogenesis of LUAD versus LUSC, we profiled their transcriptomic signatures, identified the corresponding control networks and defined key prognostic determinants of biological outcome.

Distinct gene sets have been shown to differentially associate to LUAD versus LUSC. In a study by Charkiewicz et al. [2] a 53 gene signature was identified as diagnostic between LUSC and LUAD [2]. Additional gene sets were identified by Liu et al. [3]. LUADassociated genes included tight junction and cell adhesion components. Diagnostic assessment revealed p63, TTF1, CK5/6, and Napsin A as efficient diagnostic discriminants 
for LUAD versus LUSC [4]. Other groups [5, 6] identified gene expression signatures in LUAD patient case series. Overall, though, the studies above did not provide a coherent picture of the tumor progression trajectories of NSCLC subtypes. Furthermore, no high-impact prognostic indicators were consistently identified.

We reasoned that key determinants of tumor identity were likely to be important contributors to the biological history of a tumor. Such determinants would correspondingly impact on the prognosis of distinct NSCLC subtypes. Hence, we went on to explore potential indicators of clinical outcome, by separately analyzing individual determinants associated to distinct NSCLC subtypes. This was done utilizing transcriptomic DNA microarray and associated clinical data from 2,437 NSCLC patients [7]. We examined the impact of gene expression levels in primary tumors on the progression status in NSCLC patients following surgical treatment. Transcriptomic analysis was extended with nextgeneration sequencing (NGS) of an independent NSCLC case series, to provide technology bias-independent assessment of prognostic mRNA determinants. Expression at the protein level was extensively assessed to validate all the markers utilized in the study.

Our findings led to the discovery of sets of genes that differentially determine disease outcome in distinct subgroups of lung cancers. These included key drivers of tumor growth, which were differentially involved in LUAD versus LUSC development. Our findings provide novel insight into the biological history of LUAD and LUSC and indicate that distinct tumor progression pathways are at work in the two main NSCLC subtypes.

\section{RESULTS}

\section{Prognostic determinants in LUAD versus LUSC}

Disease-associated determinants were predicted to bear on clinical outcome. We thus went on to explore the prognostic power of genes that were differentially expressed in LUSC versus LUAD. TROP2 is a widespread driver of tumor progression [8-10], and was shown to have a negative bearing on unselected cases of NSCLC (Figure 1A). Notably, though, our findings indicated that TROP2 has a vast negative prognostic impact on LUAD, but only a marginal one on LUSC, where Trop- 2 expression associates to terminal differentiation to cornified cells [11], with formation of keratin pearls (Figure 1A). Parallel findings were obtained for TP63, which we had previously shown to be both an upstream driver of Trop-2 [8,9] and a downstream effector [12]. TP63 is a powerful diagnostic discriminant [13], and a cancer prognostic [14] and predictive [15] factor. Consistent, TP63 overexpression was shown to have a strong negative impact on LUAD. However, it did associate to a trend for protection in LUSC (Figure 1B, Supplementary Table 1).
These findings raised the issue that LUSC and LUAD may follow profoundly distinct tumor progression trajectories. Hence, we went on to first systematically identify differentially expressed genes in LUAD versus LUSC, through supervised analysis of in silico datasets (Table 1, Supplementary Table 1). Then, we assessed such differentially expressed genes for impact on malignant progression of the two NSCLC subtypes [7]. A case series of breast cancer patients [16] was utilized as comparison benchmark.

Charkiewicz et al. [2] performed DNA microarray gene expression profiling in a training set of 108 NSCLC samples and a validation cohort of 44 samples [2]. This led to identify a 53 gene signature that efficiently discriminated LUSC from LUAD [2]. Additional gene sets were identified by Liu et al. [3]. Diagnostic trials identified p63, TTF1, CK5/6, and Napsin A as selectively associated to LUAD versus LUSC [4]. Chang and colleagues [6] found a 74-gene signature that discriminated LUAD versus LUSC. Lu and colleagues [5] identified a set of 16 differentially-expressed genes, as involved in the apoptotic execution phase.

The sets of differentially expressed genes identified above were parsed for redundancy and validated in silico for differential expression in LUAD versus LUSC. These genes were then systematically assessed for prognostic impact in LUAD versus LUSC, through meta-analysis of DNA microarray data from 2,437 NSCLC patients through the KMPlot database (http://www.kmplot.com). Cox regression analysis was performed and KaplanMeier (KM) survival plots were obtained. This led us to identify 69 genes (33 diagnostic for LUSC, 15 associated to LUAD, 21 which were not diagnostic for cancer type), that had significant bearing on prognosis of at least one tumor type (Supplementary Table 1). Remarkably, only $8 \%$ of LUSC-diagnostic genes and $21 \%$ of LUADdiagnostic genes showed a concordant impact on lung cancer diagnosis. Strikingly, impact profiles were more similar to benchmark breast cancers $(25 \%$ of LUAD parameters; $31 \%$ of LUSC parameters) than between NSCLC subtypes (Supplementary Table 1, Figures 2-5). Dramatic examples were those of DSG3, SERPINB13, FOXE1, GRHL3, DLX5, TMPRSS11D, TESC, which had a negative prognostic impact on LUAD, but a positive one in LUSC (Figures 4, 5A). Prognostic impact was often obscured when LUAD and LUSC were categorized together as NSCLC, e.g. in the case of JAG1, S100A1, KRT7, RPTPB, CSPG6, PDGFB (Supplementary Table 1). Further, determinants such as CLND3, TESC, which were high-risk indicators in LUAD, were detected as positive prognostic factors in unselected NSCLC (Figure 5A, Supplementary Table 1), and determinants such as ATP1B3, HPCAL3, COL4A6, SLUG, PARD6G, SOX2, $C L C A 2, S T F 1, S K P 2$, which were positive indicators in LUAD, were detected as negative prognostic factors in unselected NSCLC (Figure 5A, Supplementary Table 1). 
To validate DNA microarray findings, tumor transcriptomes were profiled by next-generation sequencing (NGS), as an orthogonal technology versus mRNA quantification by hybridization [17]. NGS analysis was conducted on series of 500 LUAD and 494 LUSC cases (https://www.proteinatlas.org/) (Supplementary Table 2). Raw data were obtained for primary assessment, and were computed as scatter plots of individual survival values (Supplementary Table 3 ).

Comparisons of NGS KM curves versus DNA microarray analysis of corresponding parameters were conducted. Concordance of prognostic impact with significant $\mathrm{P}$ values, or correspondence of lack of significant prognostic in specific subgroups were listed. A preliminary quality filter was introduced that eliminated graphs with subgroups of low numerousness $(\leq 100$ patients) and cases where the investigated parameter was not detected. Among LUSC diagnostic parameters (96 KM analyses) discordant impact was detected in 14 cases (14.6\%). Among LUAD diagnostic parameters (48 $\mathrm{KM}$ analyses) discordant impact was detected in 10 cases (20.8\%). Among parameters that were not subtype-related (63 KM analyses) discordant impact was detected in 20 cases $(31.7 \%)$. Overall, among the $207 \mathrm{KM}$ validated analyses, discordant impact was detected in 44 cases $(21.3 \%)$ for an overall, highly reliable $78.7 \%$ concordance across survival analyses.

\section{Prognostic genes with the highest impact on disease progression}

Genes whereby high versus low expression best discriminated between progressing versus nonprogressing tumors encompassed growth factor and
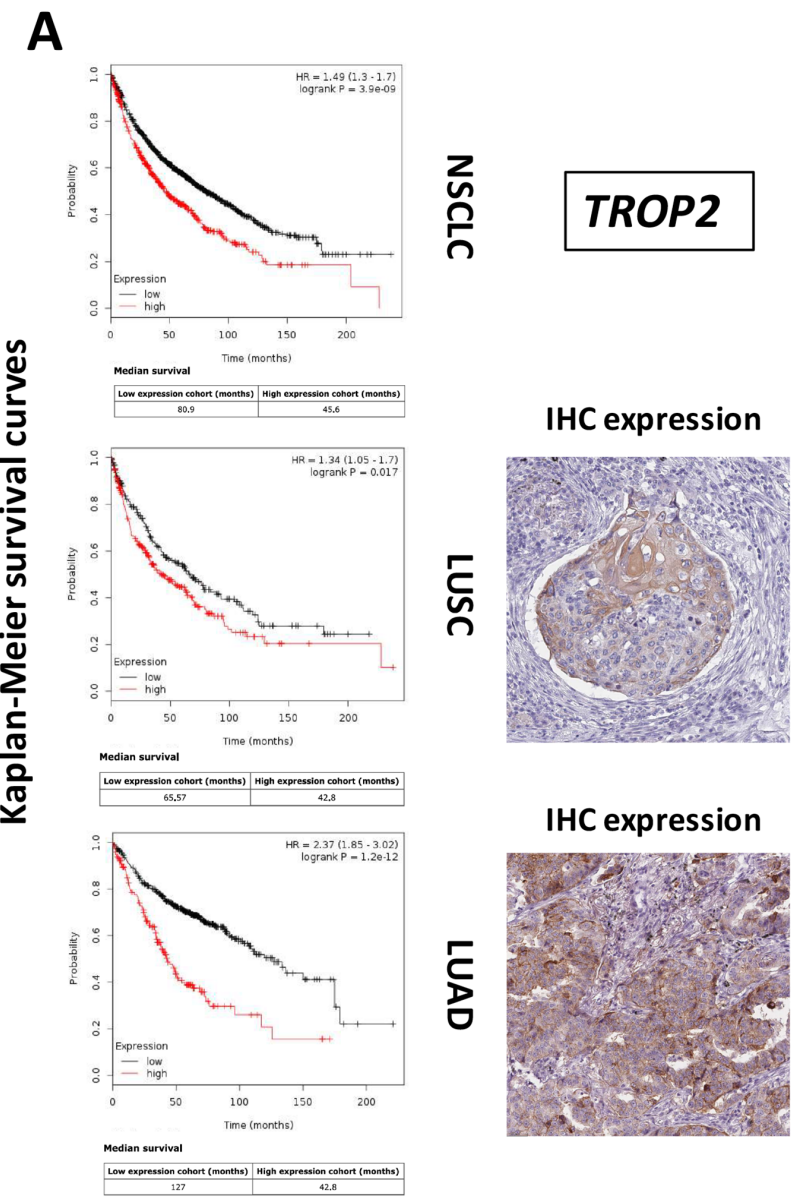

B
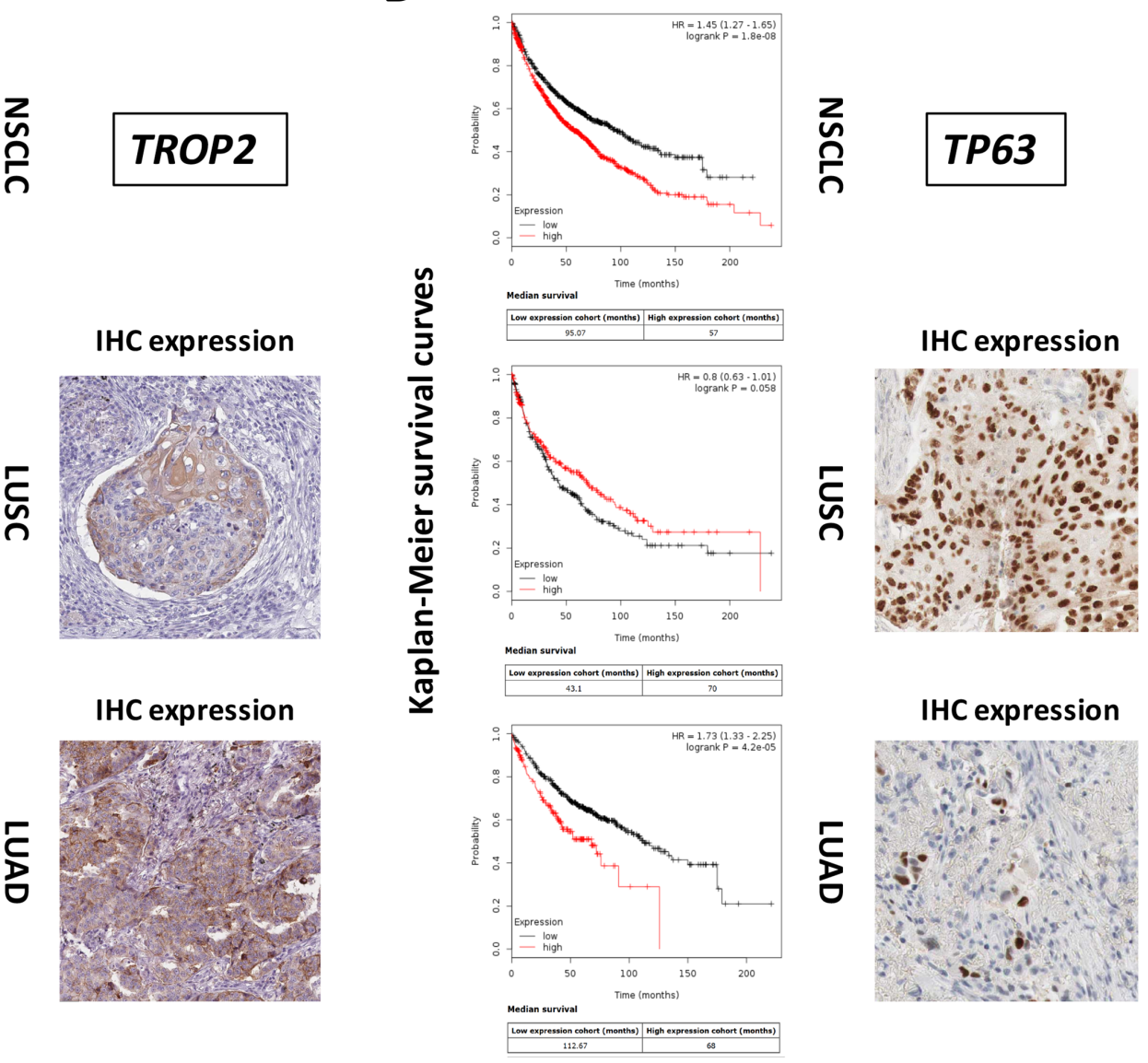

Figure 1: Differential genetic diagnostic and prognostic impact on LUAD versus LUSC. DNA microarray data from 2,437 NSCLC patients were preprocessed and meta-analyzed through the KMPlot database (http://www.kmplot.com). Gene expression data were downloaded from GEO (https://www.ncbi.nlm.nih.gov/geo/), using clinical survival information and minimum patient numbers as threshold. Databases containing high-resolution IHC images were analyzed for patterns of expression of differential diagnostic and prognostic proteins for lung LUAD versus LUSC (https://www.proteinatlas.org/). (A) KM survival curves of high (red) versus low (black) TROP2 expressors. Median survival, HR and correlated P values are indicated. (B) KM survival curves of high (red) versus low (black) TP63 expressors. Median survival, HR and correlated P values are indicated. (right side of the panels) IHC analysis of the expression of the Trop-2 or p63 proteins in LUSC or LUAD. 
Table 1: Prognostic determinants in LUAD versus LUSC

\section{LUSC prognostic determinants}

-LUSC diagnosis ${ }^{\mathrm{a}}$

-Protective factors: DSG3, SERPINB13, MRP5, FOXE1, GRHL3, DLX5

-Tumor progression determinants: SFN

-LUAD diagnosis ${ }^{b}$

-Protective factors: FOLR1, PLEKHA6

-Tumor progression determinants: SFTA3

-Not associated to diagnosis ${ }^{\mathrm{c}}$

-Protective factors: SKP2, TGFBR2

-Tumor progression determinants: MPP5, E2F

\section{LUAD prognostic determinants}

-LUSC diagnosis ${ }^{\mathrm{a}}$

-Protective factors: ATP1B3, HPCAL3, SFTA2, COL4A6, MRP5, SLUG, PARD6G, SOX2, CLCA2, RPTPB, JAG1, STF1

-Tumor progression determinants: KRT5A, KRT6A, KRT14, KRT17, PERP, SERPINB5, SERPINB13, COL7A1, DSG3, TRIM29, FGFBP1, GLUT1, SFN, TMPRS11D, FOXE1, GRHL3, PTHLH, S100A1, DLX5, ST6GALNAC2

-LUAD diagnosis ${ }^{b}$

-Protective factors: TMEM125, TTF1, TASK2, TMC5, ACSL5, FOLR1, RORC, QSOX1, SFTA3, CEACAM6, ATP11A, PLEKHA6

-Tumor progression determinants: KRT7, TESC, CLDN3

-Not associated to diagnosis ${ }^{\mathrm{c}}$

-Protective factors: GSK3B, ATR, SKP2, SMC3, CCND3, PRIM2, IGF1R, TGFBR2, CTNND1, CASK, MPP5, CTGF, PDGFA

-Tumor progression determinants: PRPF19, MCM4, MCM5, RFC2, PLK1, CDK2, PDGFB, E2F

a Utilized for LUSC diagnosis.

${ }^{b}$ Utilized for LUAD diagnosis.

${ }^{\mathrm{c}}$ Cancer drivers that are not utilized for differential diagnosis of NSCLC subtypes.

growth factor receptors, transcription factors, cell cytoskeleton and cell-cell junction components, together with constituents of the intercellular matrix (Table 1). Overexpression of the transforming growth factor (TGF) receptor 2 gene (TGFBR2) was shown to associate with less aggressive disease courses in LUSC. Correspondingly protective determinants for LUAD were $I G F 1 R$ and $P D G F A$, whereas $P D G F B$ had a negative prognostic impact on LUAD. Keratins are used as IHC markers in clinical diagnostic assays, e.g. keratin 5 and 6 for LUSC diagnosis, keratin 7 for LUAD identification. Notably, though, all overexpressed keratins, i.e. KRT5A, KRT6A, $K R T 7, K R T 14, K R T 17$, were found to play a role as LUAD tumor progression determinants. The collagen gene COL4A6 was shown to be a LUAD protective factor, whereas $C O L 7 A 1$ associated to worse prognosis. SERPINB5/maspin and SERPINB13 overexpression associated to worse disease outcome in LUAD, but showed a protective role in LUSC.

\section{Pathway regulation by prognostic genes in lung cancer}

The functional role of the identified prognostic genes was assessed versus categorized gene expression data from GEO (https://www.ncbi.nlm.nih.gov/geo/). Main pathways were found to be regulation of the cell cycle (GSK3B, ATR, SKP2, CDK1, CDK2, CDK4, SMC3, PLK1, CCND3), control of DNA replication (RFC2, PRIM2, MCM4, $M C M 5)$ and DNA repair (ATR). Cell differentiation appeared also involved, as the transcription factors TTF1, a main LUAD protective factor, is a key player in lung epithelium development. QSOX1, SOX2, SLUG, STF1 were all associated to a more benign course of disease, suggesting control of tumor initiation and cancer stemcell functions versus epithelial-mesenchimal transition in NSCLC. SOX2, and TP63 are often coamplified and their overexpression is associated to favourable disease outcome (Figures 1B, 3, 4) [18]. Cell-cell adherent junction 
LUAD

LUSC

NSCLC
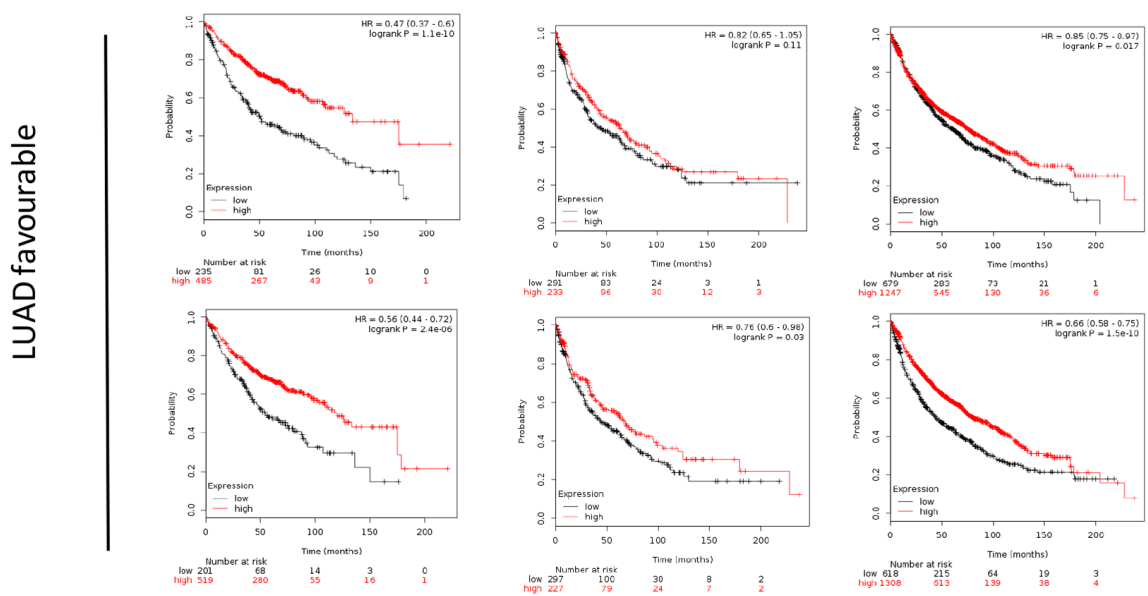

槀
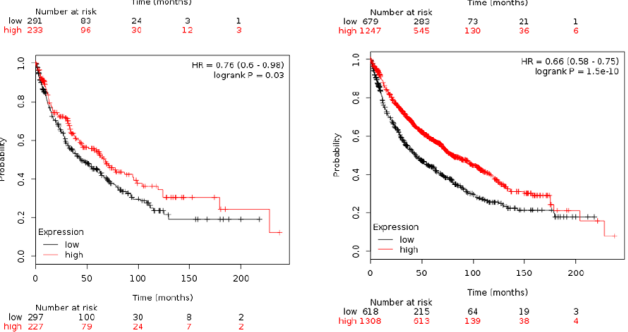

$\exists$
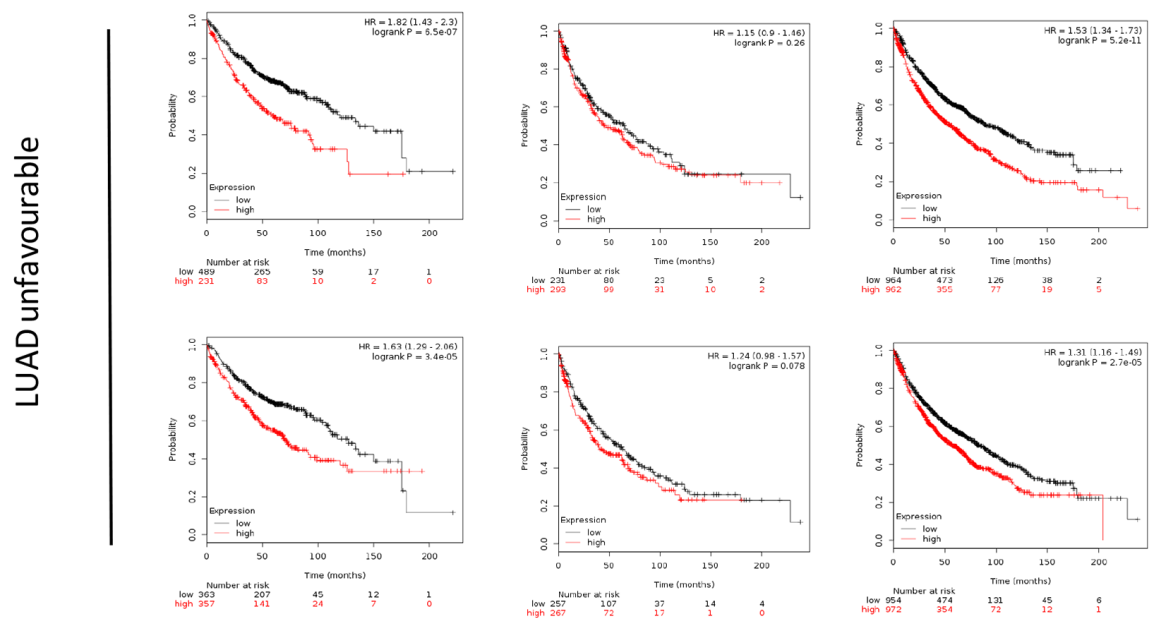

$\frac{\text { Q }}{\frac{0}{3}}$
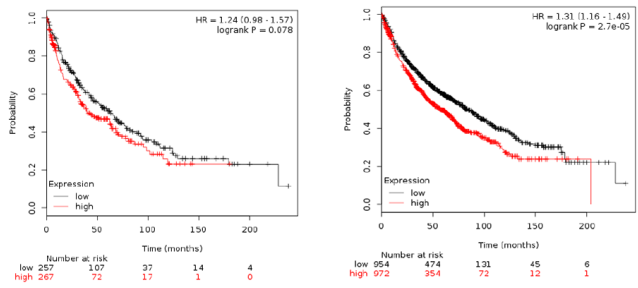

$\stackrel{n}{\circ}$
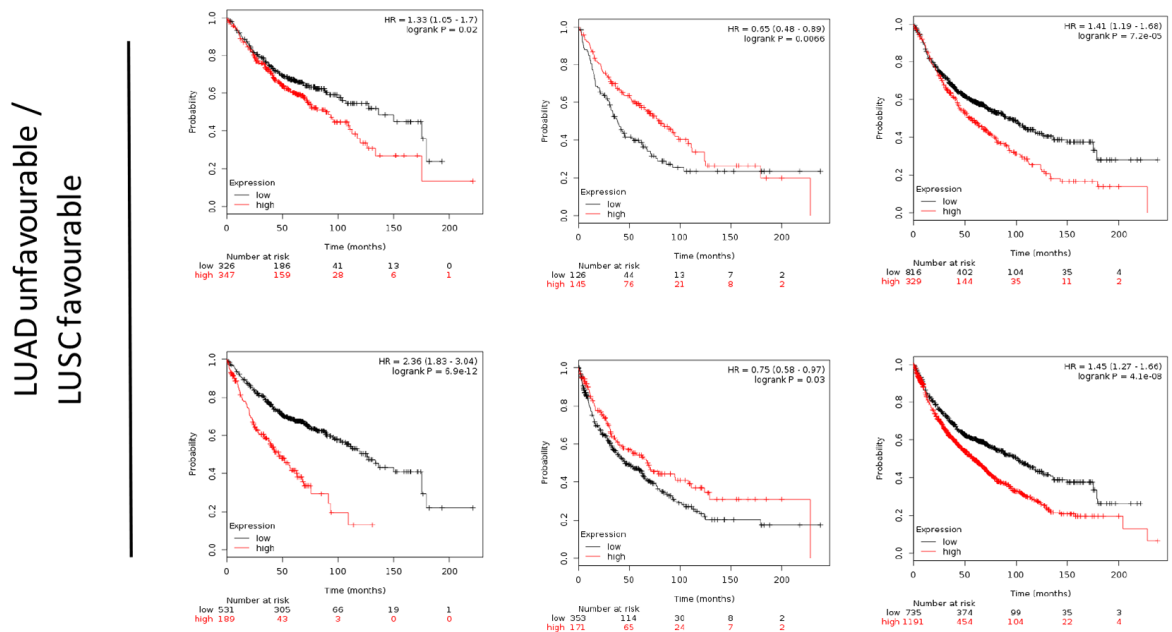

叧
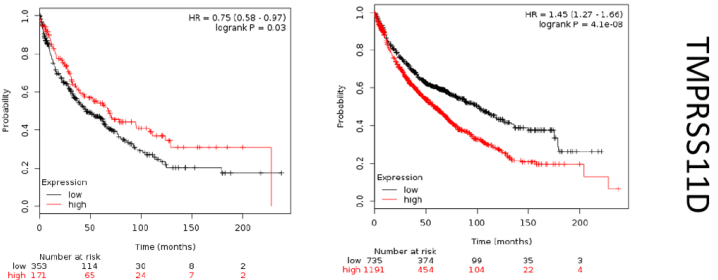

Figure 2: Survival curves of LUAD, LUSC versus unselected NSCLC. DNA microarray data from 2,437 NSCLC patients were preprocessed and meta-analyzed through the KMPlot database (http://www.kmplot.com)/GEO (https://www.ncbi.nlm.nih.gov/geo/) as described. KM survival curves of high (red) versus low (black) expressors of the genes indicated on the right are shown. Median survival, HR and correlated P values are indicated. (upper panels) favourable prognostic determinants for LUAD. (mid panels) unfavourable prognostic determinants for LUAD. (lower panels) unfavourable prognostic determinants for LUAD with positive impact on LUSC. 
components, such as CTNND1, CASK, MPP5, were associated to a better prognosis of LUAD, likely because of retained cell-cell junctions and epithelial differentiation. High levels of the transmembrane protease TMPRSS11D were previously shown to predict poor overall survival in NSCLC [19]. Our findings show that this is entirely due to the dismal outcome of expressing LUAD, as TMPRSS11D is associated to favourable prognosis in LUSC (Supplementary Table 1).

\section{Network analysis}

Whole transcriptome profiling of LUAD versus LUSC and differential prognostic analysis were utilized to reveal potential malignant progression-inducing modules, for intra-group and inter-group differentiation. Control networks involved in cell growth and apoptosis were shown to be profoundly different in LUAD versus LUSC $[3,6,8,20,21]$ (Figure 6). A pivotal p53/p63/p73 axis only emerged in LUSC (Figure 6, left panel) [20]. Additionally different molecular networks between lung LUAD and LUSC were found in the control of cell cycle, DNA repair, and metabolic pathways.

\section{Proteomic signature of the differential prognostic profiles}

Analysis conducted at the RNA level were extended to the protein level (Supplementary Table 4). In silico IHC analysis was conducted, for expression of the encoded proteins in independent cases of patients with lung cancer (Supplementary Table 4). Antibody staining validation included immunofluorescence, Western blotting, reactivity against recombinant protein and comparison of staining patterns of independently-generated antibodies (https:// www.proteinatlas.org/about/assays+annotation\#iha).

All IHC images were checked for staining intensity and fraction of stained cells. Consistent, proteins encoded by genes preferentially associated to LUAD versus LUSC were found preferentially overexpressed by the corresponding tumor types. Only a few exceptions were found, consistent with an additional layer of regulation at the protein translation level. Keratin 17 was indeed found frequently overexpressed also in LUAD, thus questioning its diagnostic role in distinguishing between the two tumor types. Corresponding findings were obtained for JAG1. FGFBP1 and ST6GALN2 proteins were found
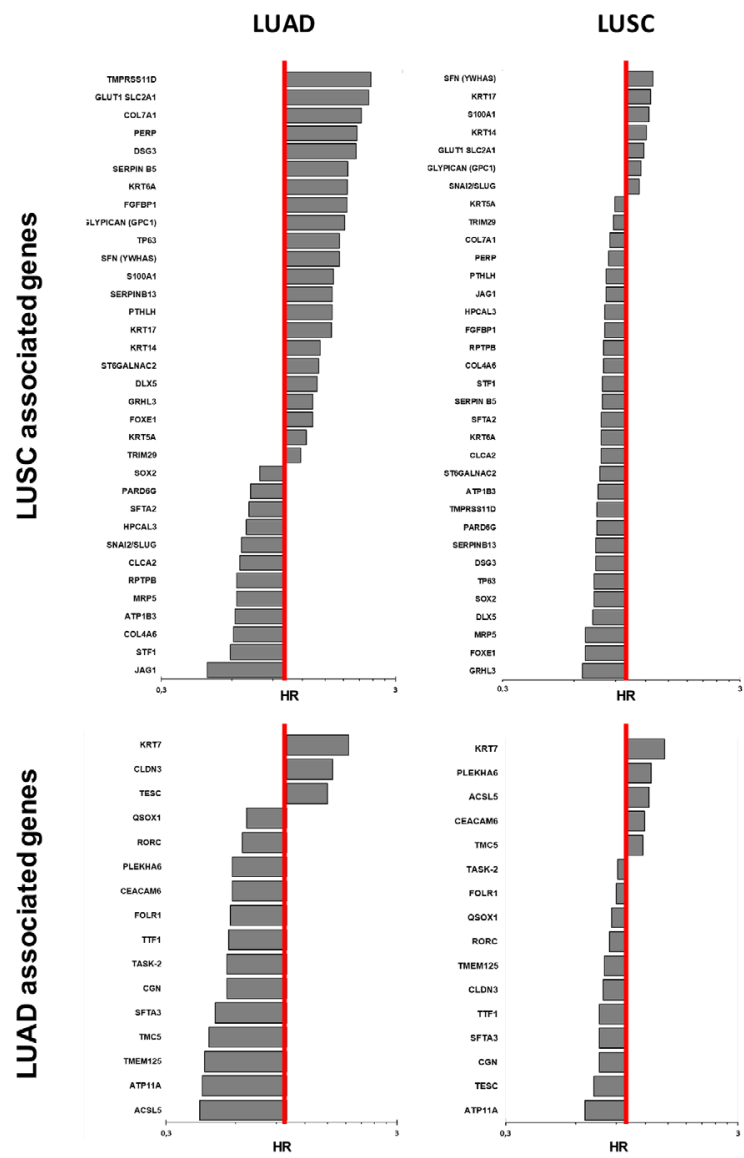
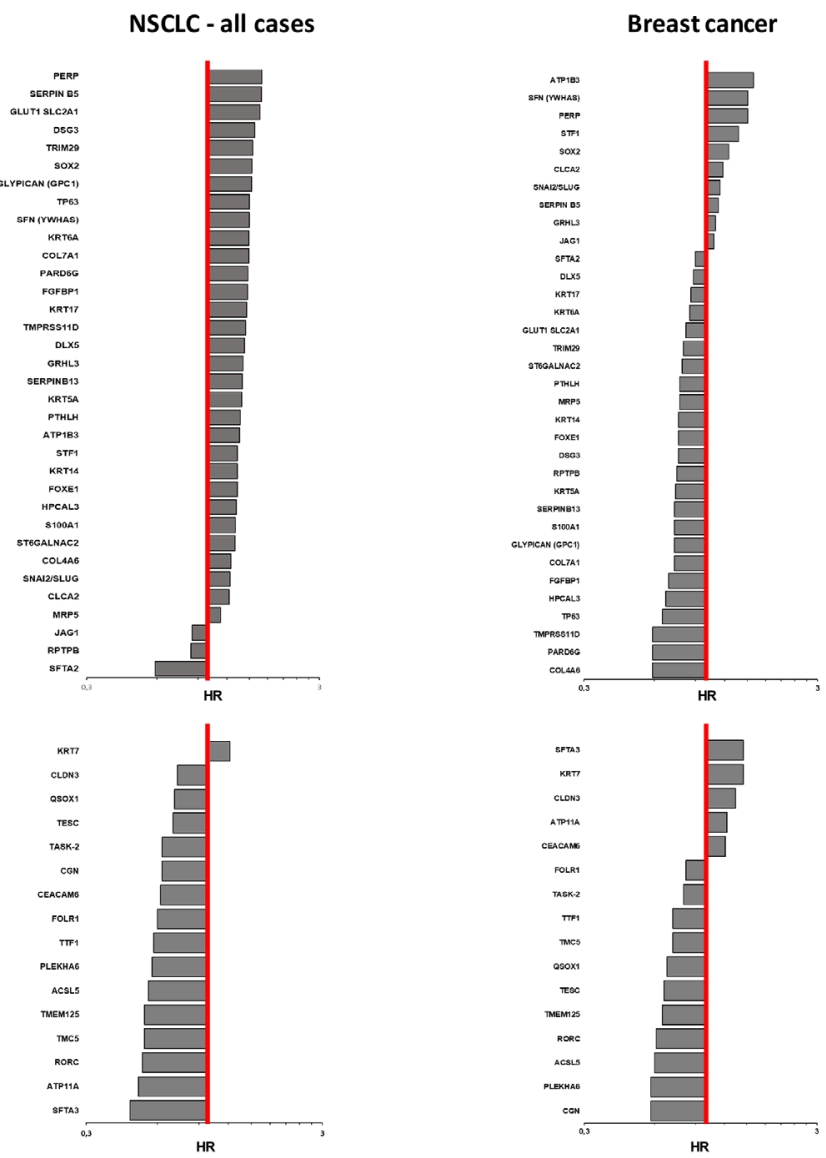

Figure 3: Quantitative impact of prognostic determinants in LUAD, LUSC and breast cancer. Bar plots show the hazard ratio (HR)/prognostic impact on overall survival of LUAD, LUSC, NSCLC and breast cancer (http://www.kmplot.com). LUSC-associated genes (upper panel) and LUAD-associated genes (bottom panel) are shown. The genes are listed in descending order of HR values in each tumor type. The red line indicates $\mathrm{HR}=1$. The bar graphs are plotted on a log scale. 
to be weakly expressed in lung cancers irrespective of tumor type.

\section{DISCUSSION}

Lung cancer is the most common cause of cancer death worldwide, with an estimated 1.6 million deaths each year [22, 23]. NSCLC are often diagnosed at an advanced stage, and the overall 5-year survival for these patients is only $15 \%-20 \%$. Particular histotypes of NSCLC may display distinct molecular characteristics and molecular determinants, which may associate with distinct histopathological and genetic characteristics of lung cancer [6]. However, current clinico-pathological staging procedures appear profoundly inadequate to dissect NSCLC into patient groups with distinct biological outcome.

Transcriptome analysis has the potential to discriminate between distinct tumor types [1]. Transcriptome analysis of independent cohorts of NSCLC cases by Charkiewicz et al. [2] led to the identification of 53 genes, that were differentially expressed in LUSC versus LUAD. Additional gene-sets were identified by Liu et al. [3]. These differentially expressed genes appeared involved in pathways related to cell proliferation, signal transduction and metabolism. Diagnostic trials identified p63, TTF1, CK5/6, and Napsin A as selectively associated to LUAD versus LUSC [4]. Chang and colleagues [6] found a 21-gene signature in the HMGB1/RAGE signaling pathway, 22 risk-modulatory genes of the ERK pathway, as triggered by beta-adrenergic receptors, and a 31-gene signature as associated to clathrin-coated vesicle recycling. Lu and colleagues [5] identified gene expression signatures in 700 LUAD cases. Among them, a set of 16 genes appeared involved in the control of the apoptotic execution phase. Transcriptional network analysis showed involvement of E2F, CTGF, and PDGF in lung cancer pathogenesis [21]. Additionally, LUSC show involvement of the EGF, IL1F8, and CX3CL1 pathways, while changes in Rb1, miR-200, and EMP2 targets appear specific for LUAD [21].

Despite these efforts, little consistency was found across independent studies [6]. Inadequate study design, small sample size and varied data analysis strategies negatively influenced study outcomes. An epitomic analysis of 47 published gene expression signatures,

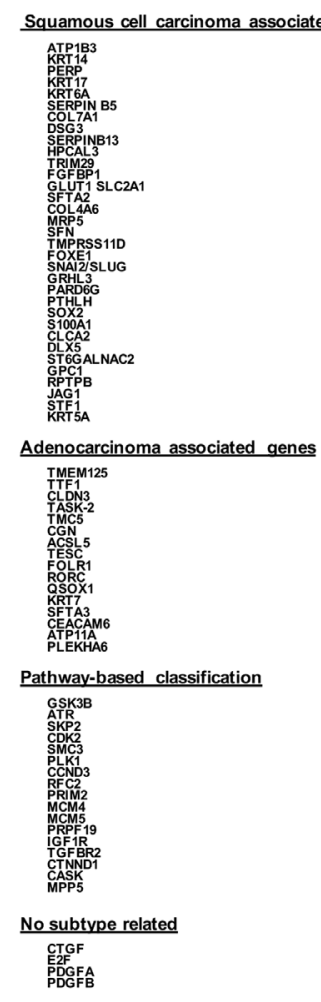

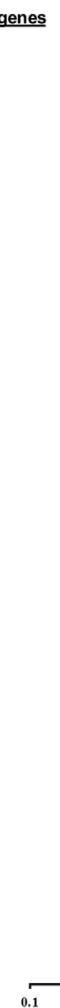

LUAD

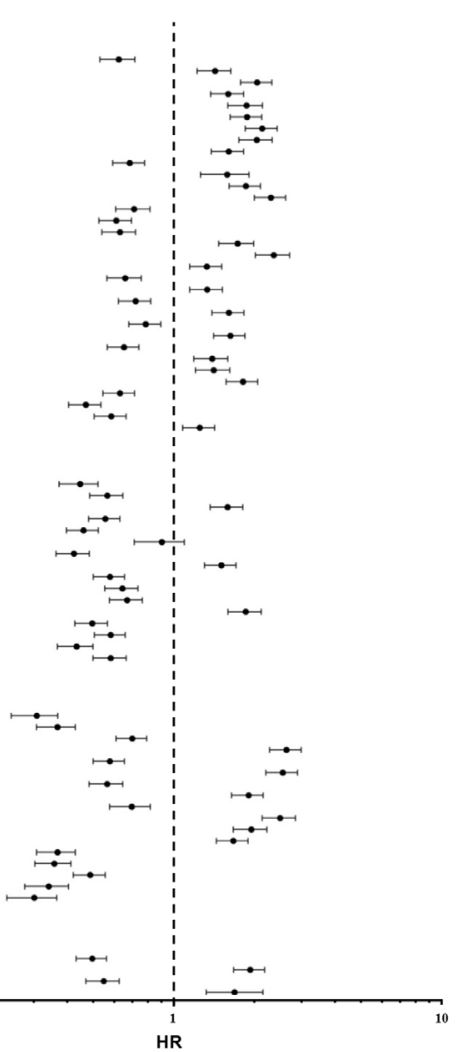

LUSC

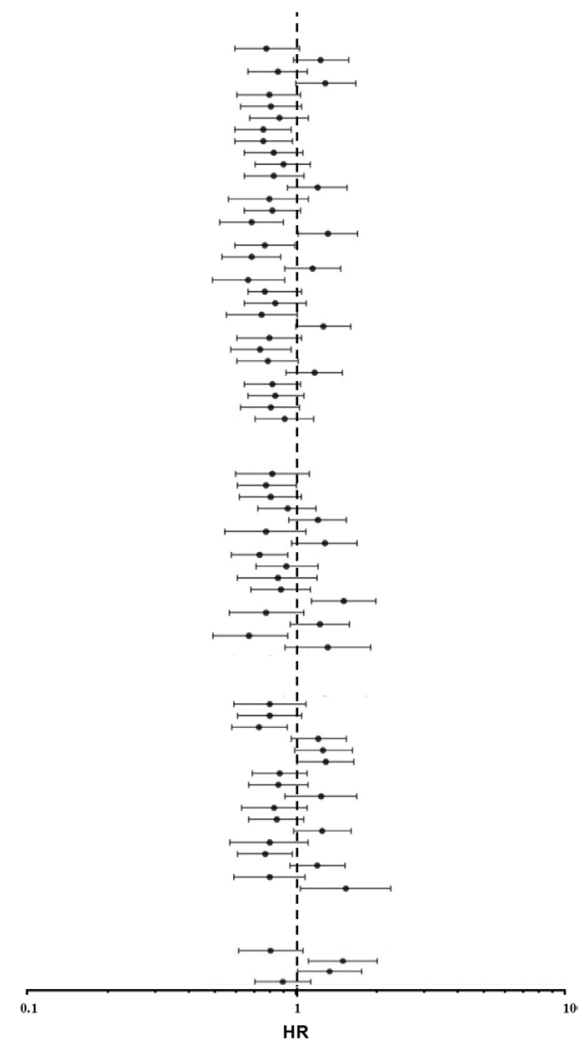

Figure 4: Distribution of disease-outcome values for prognostic LUAD versus LUSC determinants. Forest plots summarize the impact of individual determinants overexpressed in LUSC versus LUAD, with opposite prognostic impact in LUAD versus LUSC. The dashed line indicates an $\mathrm{HR}=1$. Median risk values are indicated by dots. Confidence intervals are indicated by horizontal bars. The graphs are plotted on a log scale. Comparative distribution versus undissected NSCLC is reported in Supplementary Table 1E. 


\section{A}

LUAD

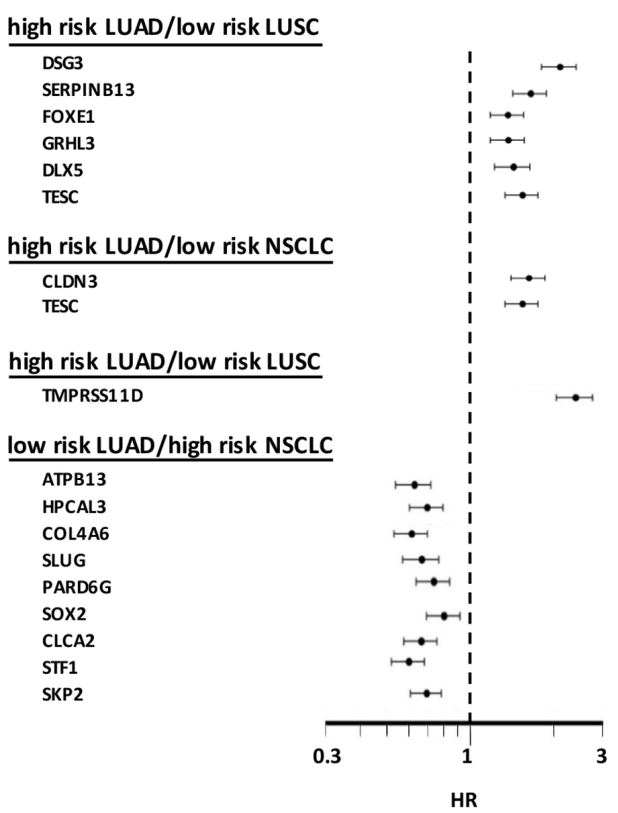

LUSC

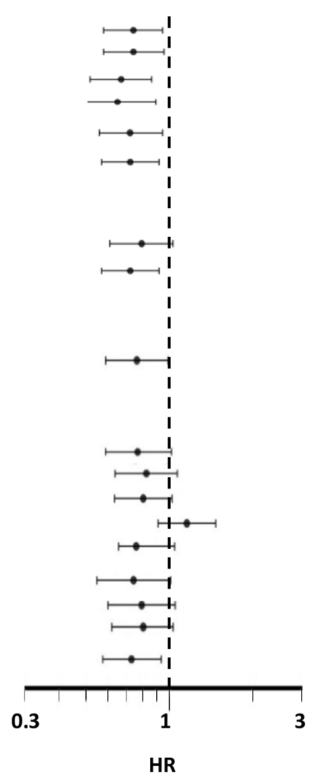

NSCLC

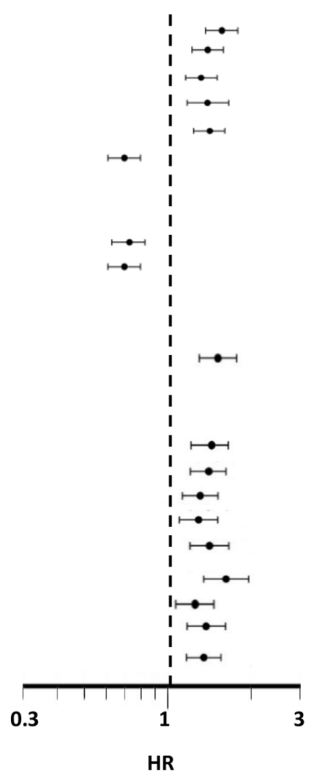

B

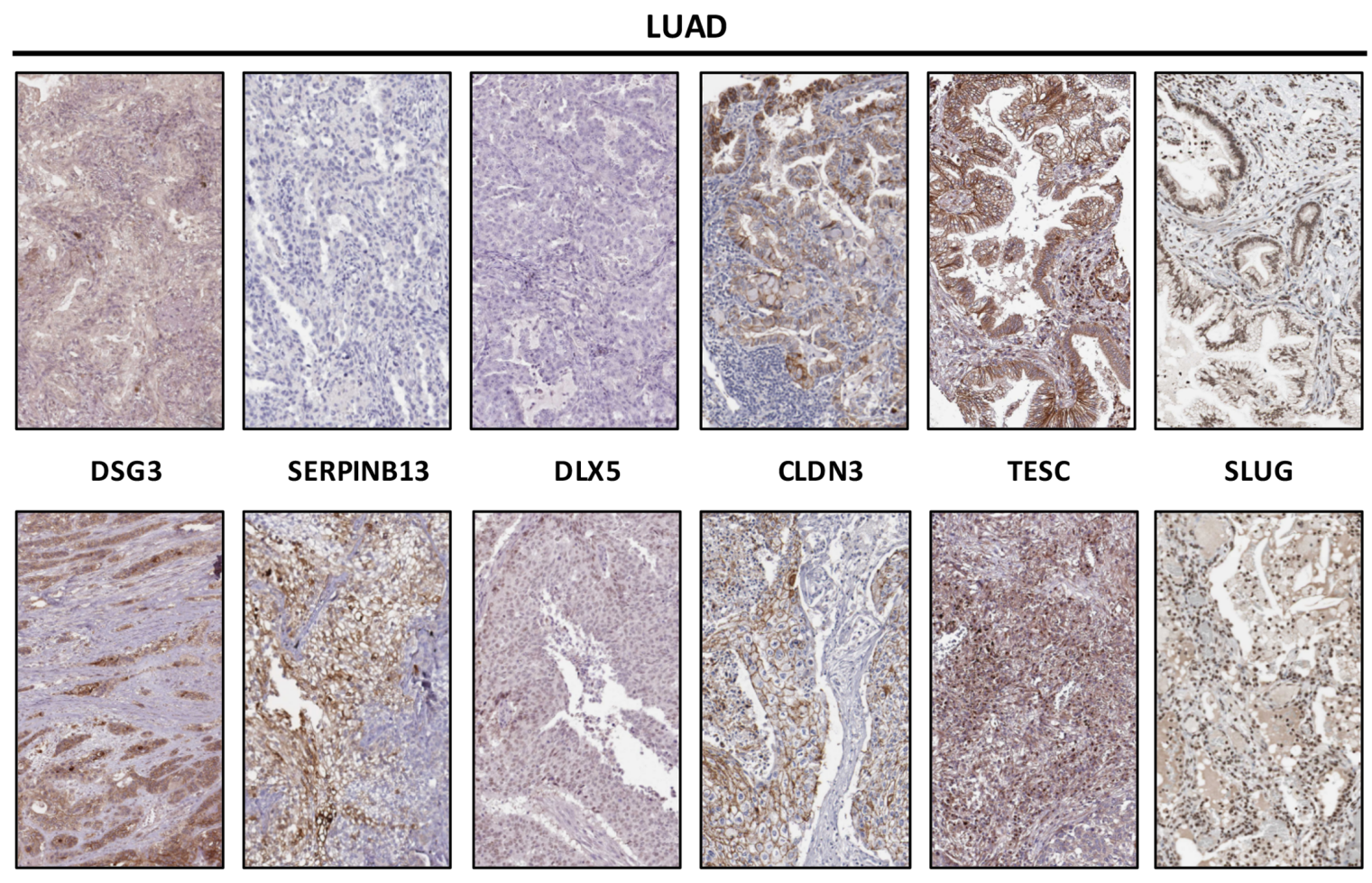

LUSC

Figure 5: Opposite prognostic determinants in LUAD versus LUSC. (A) Forest plots of the impact of individual determinants overexpressed in LUSC versus LUAD, with divergent prognostic impact on prognosis of LUAD, LUSC or NSCLC. The dashed line indicates an $\mathrm{HR}=1$. Median risk values are indicated by dots. Confidence intervals are indicated by horizontal bars. The graphs are plotted on a log scale. (B) IHC analysis of the expression of the encoded proteins of representative genes in LUSC or LUAD. Representative examples of average protein expression levels (https://www.proteinatlas.org) are reported. 
indicated that the predictive performance of several signatures was not superior to that obtained from random gene expression signatures, and sometimes performed even worse [6]. Rather unsettling, over 90\% of random signatures with more than 100 gene members appeared to bear a value as significant predictors of disease outcome [6]. Not surprisingly, to date, the only molecular traits that are of current use in clinical practice for prognostic and therapeutic purposes are genomic mutations of EGFR and KRAS and gene fusions of ALK.

We speculated that a main structural weakness of previous approaches was a blunt, overall assessment of NSCLC, as if they were a homogeneous tumor type. Categorization of divergently associated determinants was thus expected to be blurred, if not entirely lost, in such joint classification of NSCLC. Further, association to specific tumor histotypes is expected to stem from at least two distinct and opposing mechanisms. One is positive selective pressure for increased fitness and/or proliferative capacity of expressing cells. A second one is retention of differentiation traits. As such, the first mechanism is expected to be linked to malignancy, the second one, an example is Trop-2 expression terminally differentiated cornified cells in LUSC, is expected to be linked to more benign outcomes. These and additional findings supported the idea of reassessing NSCLC in a dichotomic scenario.

We thus decided to explore potential indicators of clinical outcome, by separately assessing individual determinants versus association to distinct NSCLC subtypes. This was done utilizing DNA microarray data from 2,437 NSCLC patients [7] and 3,951 control breast cancers [16]. We examined the impact of gene expression levels in primary tumors on the progression status in NSCLC patients following surgical treatment.
Distinct technologies (microarray versus next-generation sequencing versus real-time polymerase chain reaction), bear distinct limitations and detection bias, such as different probe designs and signal detection methods, or hybridization bias and thermodynamic limitations for DNA microarrays [17]. Hence, transcriptomic analysis was independently conducted through DNA microarray and NGS analysis. Large data set size, quality selection and modular cut-off values were utilized. This led us to obtain the key finding of $78.7 \%$ concordance between the two technologies. Further validation for such analyses was provided by confirmation of coherent protein expression of the analyzed genes.

Analysis for differential impact on clinical outcome then led us to discover sets of genes that differentially determine disease outcome in lung LUAD versus LUSC. These included growth factor and growth factor receptors, transcription factors, cell cytoskeleton and cell-cell junction components, together with constituents of the intercellular matrix. Overall, 69 genes were identified, that acted as prognostic determinants. Remarkably, these only had a concordant impact in $8 \%$ of LUSCdiagnostic genes, and in $21 \%$ of LUAD-diagnostic genes. Several determinants were shown to have a negative prognostic impact on LUAD, but a positive one in LUSC, such as DSG3, SERPINB13, FOXE1, GRHL3, $D L X 5$, TMPRSS11D. High-risk indicators in LUAD, were detected as positive prognostic factors in unselected NSCLC, e.g. CLND3, TESC. Low-risk indicators in LUAD, such as ATP1B3, HPCAL3, COL4A6, SLUG, $P A R D 6 G, S O X 2, C L C A 2, S T F 1, S K P 2$, were detected as negative prognostic factors in unselected NSCLC. In several other cases, prognostic impacts were simply obscured when LUAD and LUSC were categorized

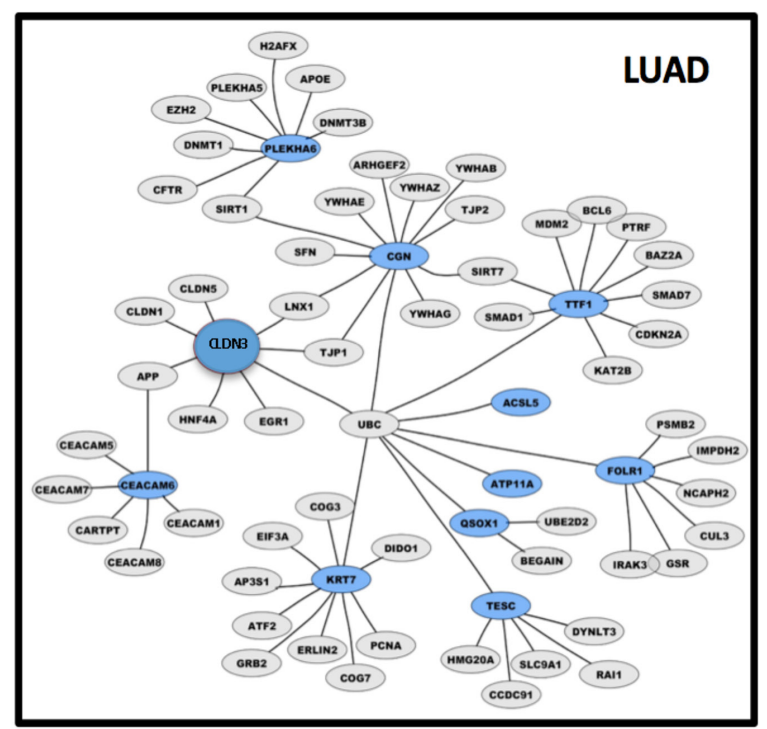

Figure 6: LUAD versus LUSC control gene networks. Graphical representation of control gene networks, as identified with Cytoscape 3.6.0. Genes are represented as nodes, biological relationships between nodes are represented as lines. Genes overexpressed in LUSC (left panel) and in LUAD (right panel) are highlighted in blue; direct interactors are in gray. 
together as NSCLC, and were lost to further prognostic analysis.

An additional result of our analysis was to help shedding light on driving signaling paths for lung cancer development. Keratin intermediate filaments play a structural role in cornified epithelia and in epidermis development [18]. Notably, all overexpressed keratins, i.e. KRT5A, KRT6A, KRT7, KRT14, KRT17, were found to play a role as LUAD tumor progression determinants. Experimental evidence had previously indicated that keratin 19 (CYFRA21-1) had a negative prognostic impact on LUAD only [24]. Thus, inappropriate expression of keratins appears as a general trait associated to LUAD aggressiveness, whether through perturbation of the differentiation status of adenocarcinoma cells or by regulation of epithelial progenitor/stem cells, as recently shown for keratin 14 [18]. The collagen gene COL4A6 was shown to be a LUAD protective factor, as possibly related to its regulatory role on cytokeratin expression and epithelial differentiation [25]. COL7A1 acted as a tumor progression determinant, possibly through its association to cancer stem cells development [26]. SERPINB5/ maspin is a putative tumor suppressors, through influence on cell-matrix interactions [27]. However, SERPINB5 disregulation occurs early during multi-step progression models of ductal pancreatic adenocarcinomas, and its overexpression associated to dismal prognosis [28], as we found in LUAD. As in LUSC, downregulation of SERPINB13 expression in head and neck squamous cell carcinomas was shown to associate with a poor differentiation grade of the tumors, presence of lymph node metastases and a decreased disease-free and overall survival [29].

Taken together, our findings thus indicate that distinct tumor progression pathways are at work in LUAD and LUSC NSCLC subtypes, and that specific determinants have a distinct impact on patient outcome, depending on tumor histology. As such, they should be taken into account in current clinical settings. This separate classification framework may correspondingly help developing and assessing novel diagnostic, prognostic and therapeutic procedures for lung cancer.

\section{MATERIALS AND METHODS}

\section{Patient case series}

For DNA microarray studies, correlated clinical and pathological data were obtained from 2,437 patients with NSCLC [7] and from 3,951 control breast cancers [16] from the Kmplot database (http:/www.kmplot. com). Samples from The Cancer Genome Atlas (TCGA) repositories (https://cancergenome.nih.gov/) were parsed versus published gene expression data and survival information. Additional data were obtained from the National Cancer Informatics Program (NCIP) (https:// cbiit.cancer.gov/ncip/ncip-home) and the Gene Expression Omnibus (GEO) (http://www.ncbi.nlm.nih.gov/geo/). For analysis of overall survival of NSCLC subgroups, lung cancer datasets were analyzed that were selected using the keywords "lung", "cancer", and related ones, and that included microarray gene expression data and correlated clinical characteristics including survival. To test for randomness, pairwise rank tests were performed for the collected clinical data, which included age, sex, smoking history, histology, stage, grade, surgery, radiotherapy and chemotherapy. For breast cancer, reference databases were established using gene expression data and survival information from 3,951 patients [16]. The median relapsefree survival was 6.43 years, $78 \%$ patients were estrogen receptor positive, and 14\% were lymph node positive. In all cases, for quantification of impact on outcome for each tested parameter, cohorts were divided into two groups, i.e. high versus low expressors, by auto selecting the best cutoff of gene expression by DNA microarray analysis. The analysis was run on selected probe set for each gene, as listed in Supplementary Table 1. Patients surviving over the selected threshold were censored instead of being excluded. In the case of breast cancer the analysis was conducted without stratification by molecular subtype and therapy [16].

NGS analysis for NSCLC was performed on TCGA data from 994 patients (494 LUSC, 500 LUAD). The NSCLC dataset included 398 females and 596 males. Most patients $(\mathrm{N}=600)$ were still alive at the time of data collection. The stage distribution was 510 stage I patients, 277 stage II patients, 163 stage III patients, 32 stage IV patients. Stage information was missing for 12 patients. NGS analysis forbreast cancer was performed on TCGA data from 1075 patients. The dataset included 1063 females and 12 males. Most patients $(\mathrm{N}=923)$ were still alive at the time of data collection. The case series included 180 stage I patients, 609 stage II patients, 243 stage III patients, 20 stage IV patients. Stage information was missing for 11 patients.

\section{DNA microarray meta-analysis}

DNA microarray data from the NSCLC and breast cancer patients were preprocessed and meta-analyzed through the KMPlot database (http://www.kmplot.com). Only Affymetrix HG-U133A (GPL96) and HG-U133 Plus 2.0 (GPL570) microarrays were considered, as they share 22,277 probe sets, to minimize variation in precision, different relative scales, and different dynamic ranges. Oligonucleotide probes for DNA array analysis were chosen for optimal hybridization to Affimetrix chips and highest signal-to-noise ratio between experimental groups. Final data were obtained through 54,675 Affymetrix probe set IDs and 70,632 gene symbols. The raw CEL files were MAS5 normalized in the R statistical environment using the Affymetrix Bioconductor library. Cox regression 
analysis, KM survival plots, hazard ratios (HR) with $95 \%$ confidence intervals, survival scatter plots and logrank $\mathrm{P}$ values were computed.

\section{NGS transcriptome profiling}

NGS transcriptomes were profiled utilizing correlated TCGA data from 994 samples from patients with lung cancer (500 LUAD; 494 LUSC) and 1075 patients with breast cancer. The TCGA RNA-seq data was mapped using the Ensembl gene id available from TCGA, and the FPKMs (number Fragments Per Kilobase of exon per Million reads) for each gene were subsequently used for quantification of expression with a detection threshold of 1 FPKM.

KM curves were obtained from analysis of correlation between mRNA expression level and patient survival. Genes with a median expression value lower than FPKM 1 were excluded. The prognosis of each group of patients was examined by KM survival estimators, and survival outcomes were compared by log-rank tests. Genes with $\log$ rank $\mathrm{P}$ values $<0.001$ in maximally separated KM analysis were classified as having prognostic impact.

\section{Immunohistochemistry}

Five $\mu \mathrm{m}$ sections from tumor samples were mounted on silanized slides. Tissue peroxidase activity was blocked with $3 \% \mathrm{H}_{2} \mathrm{O}_{2}$ for 5 minutes. Slides were quenched with $0.3 \%$ BSA in Tris-buffered saline, at room temperature, for $30 \mathrm{~min}$. For Trop-2 staining, antigen retrieval was performed by microwave treatment at $750 \mathrm{~W}$ for 10 min using $10 \mathrm{mM}$ sodium citrate buffer $\mathrm{pH} 6.0$ or $1 \mathrm{M}$ urea buffer $\mathrm{pH} 8.0$ (Dako), respectively. Slides were then incubated at room temperature for $30 \mathrm{~min}$ with the relevant antibodies. Anti-mouse (K4001, EnVision kit, Dako) and anti-goat (K0679, LSAB kit, Dako) secondary kits were used for signal amplification, as appropriate. Control sections were treated with isotypematched immunoglobulins or non-immune serum. Slides were washed in Tris-buffered saline-Tween 20, and incubated for $10 \mathrm{~min}$ in 3,3'-diaminobenzidine (DAKO). Counterstaining was performed with hematoxylin. Slides were mounted with Immunomount (Shandon). Trop-2 expression was quantified as percentage of stained cells and as intensity of the staining. An IHC score was then obtained, ranging from 0 to 12 [30]. Trop-2 expression levels were analyzed with a goat anti-Trop-2 polyclonal antibody (AF650, R\&D Systems). Antigen retrieval was performed by microwave treatment at $750 \mathrm{~W}$ for 10 min in $1 \mathrm{M}$ urea buffer (pH 8.0). The LSAB kit (K0679, Dako) was used for signal amplification. Trop-2 antigen expression was scored positive in presence of a specific staining on the tumor cell membrane, and was quantified as percentage of stained cells and as intensity of the staining. The immunostaining score (H-score) was also determined according to the following 5 categories: $0(0 \%$ of positive cells), 1 ( $<10 \%$ of positive cells), $2(10-50 \%$ of positive cells), 3 ( $50-80 \%$ of positive cells), 4 ( $>80 \%$ of positive cells). The intensity score represented the average intensity of the positive cells as follows: 1 (weak staining), 2 (moderate staining) and 3 (strong staining). The proportion and intensity scores were then multiplied to obtain the $\mathrm{H}$-score, which could range from 0 to 12 . To perform the crosstab analysis (chi-square test) between Trop-2 expression and clinicopatholigical features of patients, the protein $\mathrm{H}$-score was dichotomize using a cutoff $>4[30]$.

Databases containing high-resolution IHC images were analyzed for patterns of expression of differential diagnostic and prognostic proteins for lung LUAD versus LUSC. The Human Protein Atlas (v. 12, https:// www.proteinatlas.org/) provides spatial distribution and expression data from 16,621 proteins/21,984 antibodies and corresponding mRNA in normal human tissues and different cancer types. The expression profiles of distinctly expressed proteins in lung cancer were generated for antibody staining parameters, intensity, and fraction of positive cells in normal cells and cancers originating from different tissues [10].

\section{Gene ontology, networks, and functional impact}

Gene Ontology analysis was performed using PANTHER 7.2 software. The signaling hubs and connectivity networks were obtained using NetworkAnalyst. To condense the first-order network to its major components, a "minimum interaction network" was generated using the "Trim" function as indicated.

\section{Statistical analysis}

Statistical analysis was performed using GraphPad software (https://www.graphpad.com/). Disease free survival (DFS) was defined as the time from surgery to tumor recurrence at local or distant sites. Local relapse free survival (LRFS) and distant relapse-free survival (DRFS) were defined accordingly. KM plots were used to illustrate the survival in specified cohorts. Log-rank tests assessed equality of survival curves. SPSS software Version 15.0 was used throughout these analyses. All $\mathrm{P}$-values were two-sided.

\section{Abbreviations}

DFS: disease free survival; DRFS: distant relapsefree survival; FPKM: number fragments per kilobase of exon per million reads; HR: hazard ratios; KM: KaplanMeier; LRFS: local relapse free survival; LUAD: lung adenocarcinoma; LUSC: lung squamous cell carcinoma; NGS: next-generation sequencing; NSCLC: non-smallcell lung cancer; SCLC: small-cell lung cancer; TCGA: The Cancer Genome Atlas. 


\section{Author contributions}

V.R. and S.A. planned the study; V.R., M.T., E.G. and S.A. performed the cancer DNA microarray metaanalysis; V.R. performed NGS transcriptome profiling; V.R. and M.T. identified the gene interaction networks; all authors took part to data analysis, discussion and revision of the manuscript text.

\section{ACKNOWLEDGMENTS}

We thank R. Lattanzio for help during the course of this work.

\section{CONFLICTS OF INTEREST}

V.R. is employee and S.A. is founder and CEO of Oncoxx Biotech S.r.l.

\section{FUNDING}

The support of the Italian Ministry of Development (FESR 2016-2018). SSI000651, Region Abruzzo (POR FESR) C78C14000100005 and Oncoxx Biotech (Italian Ministry of University and Research, Smart Cities and Communities SCN_00558) is gratefully acknowledged. M.T. is supported by the Programma Per Giovani Ricercatori "Rita Levi Montalcini", Italian Ministry of University and Research (Grant PGR12I7N1Z).

\section{REFERENCES}

1. De Vita VT, Lawrence TS, Rosenberg SA. De Vita, Hellman \& Rosenberg's Cancer: Principles \& Practice of Oncology. Philadelphia: Lippincott Williams \& Wilkins; 2015.

2. Charkiewicz R, Niklinski J, Claesen J, Sulewska A, Kozlowski M, Michalska-Falkowska A, Reszec J, Moniuszko M, Naumnik W, Niklinska W. Gene Expression Signature Differentiates Histology But Not Progression Status of Early-Stage NSCLC. Transl Oncol. 2017; 10:450-58.

3. Liu J, Yang XY, Shi WJ. Identifying differentially expressed genes and pathways in two types of non-small cell lung cancer: adenocarcinoma and squamous cell carcinoma. Genet Mol Res. 2014; 13:95-102.

4. Terry J, Leung S, Laskin J, Leslie KO, Gown AM, Ionescu DN. Optimal immunohistochemical markers for distinguishing lung adenocarcinomas from squamous cell carcinomas in small tumor samples. Am J Surg Pathol. 2010; 34:1805-11.

5. Lu TP, Chuang EY, Chen JJ. Identification of reproducible gene expression signatures in lung adenocarcinoma. BMC Bioinformatics. 2013; 14:371.
6. Chang YH, Chen CM, Chen HY, Yang PC. Pathwaybased gene signatures predicting clinical outcome of lung adenocarcinoma. Sci Rep. 2015; 5:10979.

7. Győrffy B, Surowiak P, Budczies J, Lánczky A. Online survival analysis software to assess the prognostic value of biomarkers using transcriptomic data in non-small-cell lung cancer. PLoS One. 2013; 8:e82241.

8. Guerra E, Trerotola M, Aloisi AL, Tripaldi R, Vacca G, La Sorda R, Lattanzio R, Piantelli M, Alberti S. The Trop-2 signalling network in cancer growth. Oncogene. 2013; 32:1594-600.

9. Trerotola M, Cantanelli P, Guerra E, Tripaldi R, Aloisi AL, Bonasera V, Lattanzio R, de Lange R, Weidle UH, Piantelli M, Alberti S. Upregulation of Trop-2 quantitatively stimulates human cancer growth. Oncogene. 2013; 32:222-33.

10. Ambrogi F, Fornili M, Boracchi P, Trerotola M, Relli V, Simeone P, La Sorda R, Lattanzio R, Querzoli P, Pedriali M, Piantelli M, Biganzoli E, Alberti S. Trop-2 is a determinant of breast cancer survival. PLoS One. 2014; 9:e96993.

11. Alberti S, Miotti S, Stella M, Klein CE, Fornaro M, Ménard $\mathrm{S}$, Colnaghi MI. Biochemical characterization of Trop-2, a cell surface molecule expressed by human carcinomas: formal proof that the monoclonal antibodies T16 and MOv16 recognize Trop-2. Hybridoma. 1992; 11:539-45.

12. Guerra E, Trerotola M, Tripaldi R, Aloisi AL, Simeone P, Sacchetti A, Relli V, D'Amore A, La Sorda R, Lattanzio R, Piantelli M, Alberti S. Trop-2 induces tumor growth through Akt and determines sensitivity to Akt inhibitors. Clin Cancer Res. 2016; 22:4197-205.

13. Trerotola M, Rathore S, Goel HL, Li J, Alberti S, Piantelli M, Adams D, Jiang Z, Languino LR. CD133, Trop-2 and alpha2beta1 integrin surface receptors as markers of putative human prostate cancer stem cells. Am J Transl Res. 2010; 2:135-44.

14. Biganzoli E, Coradini D, Ambrogi F, Garibaldi JM, Lisboa P, Soria D, Green AR, Pedriali M, Piantelli M, Querzoli P, Demicheli R, Boracchi P, Nenci I, et al. p53 status identifies two subgroups of triple-negative breast cancers with distinct biological features. Jpn J Clin Oncol. 2011; 41:172-79.

15. Cimoli G, Malacarne D, Ponassi R, Valenti M, Alberti S, Parodi S. Meta-analysis of the role of p53 status in isogenic systems tested for sensitivity to cytotoxic antineoplastic drugs. Biochim Biophys Acta. 2004; 1705:103-20.

16. Györffy B, Lanczky A, Eklund AC, Denkert C, Budczies J, Li Q, Szallasi Z. An online survival analysis tool to rapidly assess the effect of 22,277 genes on breast cancer prognosis using microarray data of 1,809 patients. Breast Cancer Res Treat. 2010; 123:725-31.

17. Carletti E, Guerra E, Alberti S. The forgotten variables of DNA array hybridization. Trends Biotechnol. 2006; 24:443-48.

18. Sánchez-Danés A, Blanpain C. Deciphering the cells of origin of squamous cell carcinomas. Nat Rev Cancer. 2018; 18:549-61. 
19. Cao X, Tang Z, Huang F, Jin Q, Zhou X, Shi J. High TMPRSS11D protein expression predicts poor overall survival in non-small cell lung cancer. Oncotarget. 2017; 8:12812-19. https://doi.org/10.18632/oncotarget.14559

20. Campbell JD, Yau C, Bowlby R, Liu Y, Brennan K, Fan H, Taylor AM, Wang C, Walter V, Akbani R, Byers LA, Creighton CJ, Coarfa C, et al. Genomic, Pathway Network, and Immunologic Features Distinguishing Squamous Carcinomas. Cell Rep. 2018; 23:194-212.e6.

21. Daraselia N, Wang Y, Budoff A, Lituev A, Potapova O, Vansant G, Monforte J, Mazo I, Ossovskaya VS. Molecular signature and pathway analysis of human primary squamous and adenocarcinoma lung cancers. Am J Cancer Res. 2012; 2:93-103.

22. Rotow J, Bivona TG. Understanding and targeting resistance mechanisms in NSCLC. Nat Rev Cancer. 2017; 17:637-58.

23. Herbst RS, Morgensztern D, Boshoff C. The biology and management of non-small cell lung cancer. Nature. 2018; 553:446-54.

24. Nisman B, Amir G, Lafair J, Heching N, Lyass O, Peretz T, Barak V. Prognostic value of CYFRA 21-1, TPS and CEA in different histologic types of non-small cell lung cancer. Anticancer Res. 1999; 19:3549-52.

25. Komori T, Ono M, Hara ES, Ueda J, Nguyen H, Nguyen H, Yonezawa T, Maeba T, Kimura-Ono A, Takarada T,
Momota R, Maekawa K, Kuboki T, et al. Type IV collagen alpha 6 chain is a regulator of keratin 10 in keratinization of oral mucosal epithelium. Sci Rep. 2018; 8:2612.

26. Oktem G, Sercan O, Guven U, Uslu R, Uysal A, Goksel G, Ayla S, Bilir A. Cancer stem cell differentiation: TGF $\beta 1$ and versican may trigger molecules for the organization of tumor spheroids. Oncol Rep. 2014; 32:641-49.

27. Teoh SS, Whisstock JC, Bird PI. Maspin (SERPINB5) is an obligate intracellular serpin. J Biol Chem. 2010; 285:10862-69.

28. Cao D, Zhang Q, Wu LS, Salaria SN, Winter JW, Hruban RH, Goggins MS, Abbruzzese JL, Maitra A, Ho L. Prognostic significance of maspin in pancreatic ductal adenocarcinoma: tissue microarray analysis of 223 surgically resected cases. Mod Pathol. 2007; 20:570-78.

29. de Koning PJ, Bovenschen N, Leusink FK, Broekhuizen R, Quadir R, van Gemert JT, Hordijk GJ, Chang WS, van der Tweel I, Tilanus MG, Kummer JA. Downregulation of SERPINB13 expression in head and neck squamous cell carcinomas associates with poor clinical outcome. Int $\mathrm{J}$ Cancer. 2009; 125:1542-50.

30. Spizzo G, Obrist P, Ensinger C, Theurl I, Dünser M, Ramoni A, Gunsilius E, Eibl G, Mikuz G, Gastl G. Prognostic significance of Ep-CAM AND Her-2/neu overexpression in invasive breast cancer. Int J Cancer. 2002; 98:883-88. 